Revista X é uma publicação semestral editada pelo Departamento de Letras Estrangeiras Modernas e vinculada ao Programa de Pós-Graduação em Letras, da Universidade Federal do Paraná.

ISSN:1980-0614

EDITORES-CHEFE

Dra. Juliana Zeggio Martinez (UFPR)

Dr. Alison Roberto Gonçalves (UFPR)

EDITORAS ASSOCIADAS

Dra. Ana Paula Marques Beato-Canato (UFPR)

Dra. Denise Akemi Hibarino (UFPR)

Dra. Nylcéa Thereza de Siqueira Pedra (UFPR)

\title{
COMISSÃO EDITORIAL
}

Dra. Clarissa Menezes Jordão (UFPR)

Dra. Denise Cristina Kluge (UFRJ)

Dr. Francisco Carlos Fogaça (UFPR)

Dr. Lynn Mario Trindade Menezes de Souza (USP)

Dr. John Corbett (University of Glasgow)

Dr. Kanavillil Rajagopalan (UNICAMP)

Dr. Neil Basner (University of Winnipeg)

Dra. Tania Regina Romero (UFLA)

Dra. Telma Nunes Gimenez (UEL)

LAYOUT E ELABORAÇÃO DA CAPA: Maria Teresa Ferreira Lima de Almeida DIAGRAMAÇÃO: Juliana Zeggio Martinez REVISÃO TEXTUAL:

Fernanda Cristina Lopes

João Victor Schmicheck

NORMALIZAÇÃO:

Helena Grassi

Rogério Back

Tainara Dos Santos Alexandre

Gestão Atual:

Reitor: Ricardo Marcelo Fonseca

Vice-Reitora: Graciela Inês Bolzón de Muniz 
Pró-Reitor de Graduação: Eduardo Salles de Oliveira Barra

Diretora do Setor de Ciências Humanas: Lígia Negri

Vice-diretor do Setor de Ciências Humanas: Rodrigo Rodriguez Tavares

Chefe do Departamento de Letras Estrangeiras Modernas: Anna Beatriz da Silveira Paula

Coordenação do Curso de Letras

Coordenador: Guilherme Gontijo Flores

Vice-Coordenadora: Viviane Araújo Alves da Costa Pereira

Departamento de Letras Estrangeiras Modernas

Rua General Carneiro, 460 - $9^{\circ}$ andar

80060-150 Curitiba - Brasil

Telefone [41] 3360-5183

E-mail: secretaria.delem@gmail.com

Programa de Pós-Graduação em Letras

Rua General Carneiro, 460 - $10^{\circ}$ andar

80060-150 Curitiba - Brasil

Telefone [41] 3360-5102

E-mail: ppgglet@gmail.com

Catalogação na publicação

Fernanda Emanoéla Nogueira - CRB 9ª/1607

Biblioteca de Ciências Humanas - UFPR

Revista X [recurso eletrônico]. / UFPR. Departamento de Letras Estrangeiras Modernas; editores-chefes: Juliana Zeggio Martinez, Alison Roberto Gonçalves; layout e elaboração da capa: Maria Teresa Ferreira Lima de Almeida; diagramação: Juliana Zeggio Martinez. - v.1, n.1 (jan./jun.) 2006. - Curitiba, PR : Departamento de Letras Estrangeiras Modernas -UFPR, 2020.

v.15, n.7, jul-dez. 2020

ISSN : 1980 - 0614

Disponível em : https://revistas.ufpr.br/revistax/

1. Linguística aplicada - Periódicos. I. Departamento de Letras Estrangeiras Modernas. II. Martinez, Juliana Zeggio. III. Gonçalves, Alison Roberto. IV. Almeida, Maria Teresa Ferreira Lima de. V. Titulo.

CDD -410 